\title{
The Characterization of Rational Numbers Belonging to a Minimal Path in the Stern-Brocot Tree According to a Second Order Balancedness
}

\author{
Andrea Frosini ${ }^{1}$ and Lama Tarsissi ${ }^{2,3(\otimes)}$ \\ 1 Dipartimento di Matematica e Informatica, Università di Firenze, \\ Viale Morgagni 65, 50134 Firenze, Italy \\ andrea.frosini@unifi.it \\ 2 LAMA, Univ Gustave Eiffel, UPEC, CNRS, 77454 Marne-la-Vallée, France \\ 3 LIGM, Univ Gustave Eiffel, CNRS, ESIEE Paris, 77454 Marne-la-Vallée, France \\ lama.tarsissi@esiee.fr
}

\begin{abstract}
In 1842, Dirichlet observed that any real number $\alpha$ can be obtained as the limit of a sequence $\left(\frac{p_{n}}{q_{n}}\right)$ of irreducible rational numbers. Few years later, M. Stern (1858) and A. Brocot (1861) defined a treelike arrangement of all the (irreducible) rational numbers whose infinite paths are the Dirichlet sequences of the real numbers and are characterized by their continued fraction representations. The Stern-Brocot tree is equivalent to the Christoffel tree obtained by ordering the Christoffel words according to their standard factorization. We remark that the Fibonacci word's prefixes belong to a minimal path in the Christoffel tree with respect to the second order balancedness parameter defined on Christoffel words. This alows us to switch back to the Stern-Brocot tree, in order to give a characterization of the continued fraction representation for all the rational numbers belonging to minimal paths with respect to the growth of the second order balancedness.
\end{abstract}

Keywords: Continued fractions $\cdot$ Stern-Brocot tree $\cdot$ Christoffel words $\cdot$ Balance property $\cdot$ Minimal path

\section{Introduction}

It appears that balancedness [2] is a crucial notion for the recently developed research area of combinatorics on words. A widely studied class of binary balanced words are the Christoffel words, whose interest arises from their strong connections with geometry, algebra, and number theory (see [2] for the definitions, first properties and extensive bibliography). Each Christoffel word is the

\footnotetext{
This work was partly funded by the French Programme d'Investissements d'Avenir (LabEx Bézout, ANR-10-LABX-58) and ANR-15-CE40-0006. 
discretization of a line segment of rational slope, and it can be uniquely factorized into two different Christoffel words. Such standard factorization allows us to arrange all the Christoffel words in a binary tree structure, called Christoffel tree, whose root is the word $w=01=(0,1)$. The notion of balancedness allows us to define, for each binary word $w$, a balance matrix $B_{w}$ whose maximal element provides its order of balancedness, noted by $\delta(w)$. The present study focuses on Christoffel words that are balanced words, i.e. whose order of balancedness is 1 . In this case, each row of the balance matrix is again a binary word, whose order of balancedness can be computed again, obtaining a second order balance matrix $U_{w}$ whose maximal element is defined as the second order balance value $\delta^{2}(w)$. This value can also be obtained using the link between the abelian complexity and balanced words represented by Zamboni ([6], Section 4), using the Parikh vectors.

The matrix $U_{w}$ has several properties that allow a recursive construction by referring to three $U_{w^{\prime}}$ matrices, of some specific ancestors $w^{\prime}$ of $w$ in the Christoffel tree [12]. The authors used this notion to study the distribution of $1^{\prime} \mathrm{s}$ in Christoffel words and relate it to the longstanding problem of their synchronization $[7,8]$.

The correspondence between Christoffel word and continued fraction representation of its rational slope allows us to transfer the notion of balancedness to the Stern-Brocot tree. This tree, indicated hereafter as SB-tree, is a binary tree where the irreducible rational numbers are arranged according to a specific operator called Farey sum, defined independently in [4] and in [11], and that perfectly mimes the notion of Standard decomposition of a Christoffel word. In our study, we first establish a connection between the second order balance value of a Christoffel word and the form of the continued fraction representation of the slope of the related discrete segment. Then, we present the sequence of the ratios of consecutive Fibonacci numbers constitutes, in the SB-tree, as being an example of a path which minimizes the growth of the second order balancedness parameter of the related Christoffel words. Finally, relying on this result, we characterize the minimal paths (w.r.t the second order balance value) by mixing algebraic and arithmetic techniques on the continued fraction representations. In fact, in the final theorem, we determine the rational numbers belonging to a minimal path with respect to the form of their continued fractions.

The paper is structured as follows: in Sect.2, we recall the definitions of Christoffel words, Christoffel tree and the Stern-Brocot tree. In Sect. 2.4, we introduce the notion of balancedness and we define the balance matrix of a binary word. Then we generalize the notion to the second order balance matrix. Finally, in Sect.3, we show how the second order balancedness parameter is spread on the SB-tree, providing the example of the Fibonacci sequence. Here we provide our main result: the characterization of the minimal paths in the SB-tree according to the $\delta^{2}$ parameter. We prove that a rational number on the SB-tree belongs to a minimal path, according to the growth of a second order balancedness, if the elements of its continued fractions start by 0,1 and end by 2 , while the middle terms are only made of blocks of $(1,1,1) ;(2,1) ;(1,2)$ or $(3)$. 


\section{Definitions and Previous Results}

We refer to the book [5] for the standard terminology in combinatorics of words: alphabet, word, length of a word, occurrence of a letter, factor, prefix, suffix, period, conjugate, primitive, reversal, palindrome etc. The related notation will be recalled when used.

Christoffel Paths and Christoffel Words. In discrete geometry, the theory of Christoffel words has been considered during this last few decades and has acquired a prominent role in the study of the discretization of segments and shapes. Concerning the former, let $a, b$ be two co-prime numbers; the Christoffel path of slope $\frac{a}{b}$ is defined as the connected path joining the origin $O(0,0)$ to the point $(b, a)$ in the integer lattice $\mathbb{Z} \times \mathbb{Z}$; such that it is the nearest path below the Euclidean line segment joining these two points, as shown in Fig. 1. So, there are no points of the discrete plane between the path and the line segment.

Still in Fig. 1, we can see the coding of a Christoffel path by a binary word, say Christoffel word, whose letters 0 and 1 represent a horizontal and a vertical step in the path, respectively.

The Christoffel word related to the path reaching the point $(b, a)$ is indicated by $C\left(\frac{a}{b}\right)$, and its slope is $\frac{a}{b}=\frac{|w|_{1}}{|w|_{0}}$, where the notation $|w|_{x}$ stands for the number of occurrences of the letter $x$ in $w$.

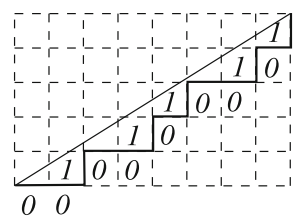

Fig. 1. The Christoffel path of the line segment of slope $\frac{5}{8}$, and the corresponding Christoffel word $C\left(\frac{5}{8}\right)=0010010100101$.

A binary word $w$ is $k$-balanced, with $k>0$, if, for any two factors $u$ and $v$ of its conjugates such that $|u|=|v|$, it follows that $\left.|| u\right|_{1}-|v|_{1} \mid \leq k$. One of the most important properties of Christoffel words is that they are 1-balanced binary words, or simply balanced words, as shown in [3].

\subsection{Christoffel Tree}

Let us recall the definition of the Christoffel tree, i.e. a well-known arrangement of the Christoffel words as a binary tree (see [1]). The root of the Christoffel tree is labeled by the pair $(0,1)$, representing the Christoffel word 01 of slope $\frac{1}{1}$. Each node $(u, v)$ generates two children according to two functions $\phi_{0}$ and $\phi_{1}$ such that: $\phi_{0}(u, v)=(u, u v)$ produces the left-child, and $\phi_{1}(u, v)=(u v, v)$ 
produces the right-child. Each node $(u, v)$ corresponds to the Christoffel word $u v$ and represents its standard factorization form [5]. Figure 2 shows the first levels of the Christoffel tree.

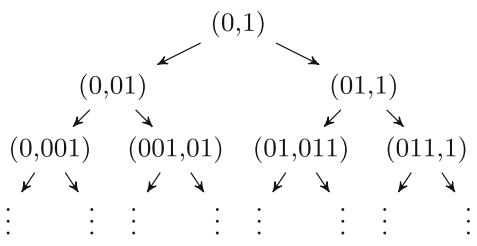

Fig. 2. The first levels of the Christoffel tree.

Let $w=C\left(\frac{a}{b}\right)$ be a Christoffel word lying at level $k$ of the tree. The directive sequence of $w$ is the word $\Delta\left(\frac{a}{b}\right)=i_{1} i_{2} \ldots i_{k}$ such that $w=\left(\phi_{i_{k}} \circ \ldots \circ \phi_{i_{2}} \circ\right.$ $\left.\phi_{i_{1}}\right)(0,1)$. According to the definition of $\phi_{0}$ and $\phi_{1}$, the elements of $\Delta\left(\frac{a}{b}\right)$ also show, step by step, the directions to reach the word $w$ in the tree starting from its root at level 1 . If $i_{n}=0$, we must move to the left, otherwise, to the right.

As an example, the directive sequence of the Christoffel word $C\left(\frac{4}{3}\right)=0101011$ at level 4 of the Christoffel tree is $\Delta\left(\frac{4}{3}\right)=100$ : according to the definition it holds $\left(\phi_{0} \circ \phi_{0} \circ \phi_{1}\right)(0,1)=\left(\phi_{0} \circ \phi_{0}\right)(01,1)=\phi_{0}(01,011)=(01,01011)$.

\subsection{Stern-Brocot Tree}

The Christoffel tree is known to be isomorphic to the SB-tree, that was introduced by M. Stern [11] and A. Brocot [4] as a binary-tree arrangement of the irreducible fractions. Such arrangement relies on the Farey sum operator, indicated by $\oplus$, and defined on two generic rational numbers as $\frac{a}{b} \oplus \frac{c}{d}=\frac{a+c}{b+d}$. The root of the SB-tree is labeled with the fraction $\frac{1}{1}$ and each node at level $n>1$ is labeled with the Farey sum of its nearest left and right ancestors, i.e., the nodes lying on the greatest level of the tree and having $k$ in its left and right subtree, respectively. The left and right ancestors of the root are considered to be the fractions $\frac{0}{1}$ and $\frac{1}{0}$, even if not present in the tree. The first levels of the SB-tree are depicted in Fig. 3.

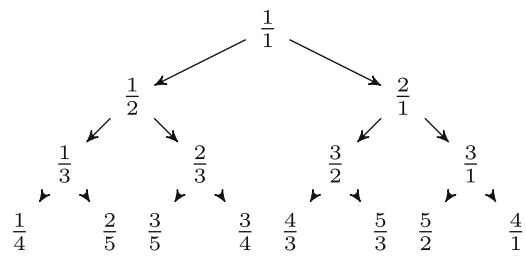

Fig. 3. The first levels of the Stern-Brocot tree. 
As an example, we compute the left-child and right-child of the node $\frac{3}{4}$ by Farey summing it with its left-ancestor $\frac{2}{3}$ and with its right-ancestor $\frac{1}{1}$, and obtaining $\frac{5}{7}$ and $\frac{4}{5}$, respectively. The SB-tree contains once each irreducible fraction and over the years has attracted researchers for its many interesting properties.

Remark 1. By the construction of the SB-tree, the following hold

i) The tree is divided into two symmetric parts. The left part contains all the reduced fractions less than 1 , while the right part contains the reduced fractions bigger than 1 .

ii) For each level $k$ of the SB tree, the rational numbers appear in increasing order from left to right, and their values are bounded by $\frac{1}{k}$ and $\frac{k}{1}$.

From our perspective, the SB-tree's appeal reveals in its close relationship with the Christoffel tree: in fact, we can note that if we replace the label of each node $(u, v)$ of this last with the rational number $\frac{|u v|_{1}}{|u v|_{0}}$ representing the slope of the corresponding Christoffel word, we obtain the SB-tree. This correspondence is interesting but not surprising once we observe that the slope of the concatenation of two Christoffel words is the Farey sum of their slopes. For instance, take the rational number $\frac{3}{5}$ on the SB-tree, it is at the same position of the Christoffel word 00100101 of slope $\frac{3}{5}$ on the Christoffel tree.

\subsection{Continued Fraction Representation}

Our study requires to recall a final notion to identify the nodes of the SB-tree. Let $\frac{a}{b}$ be a positive fraction, we define its continued fraction representation as the sequence of integers $\left[a_{0}, \ldots, a_{z}\right]$, with $a_{0} \geq 0$, represented below, and such that for each $1 \leq i \leq z, a_{i} \geq 1$ :

$$
\frac{a}{b}=a_{0}+\frac{1}{a_{1}+\frac{1}{\cdots \frac{1}{a_{z-1}+\frac{1}{a_{z}}}}}
$$

In order to obtain a unique continued fraction representation of each rational number, it is also commonly required that if $z \geq 2$, then $a_{z} \geq 2$. Otherwise, with a simple calculation, we can remark that if $a_{z}=1$, it is sufficient to reduc ethe sequence to $a_{z-1}$ and add +1 , for example: $\frac{2}{3}=[0,1,1,1]=[0,1,2]$. The continued fraction representation of a rational number is always finite while the representation is infinite in the case of an irrational number (among the vast literature on continued fraction representation, we refer the reader to [10] for the main properties).

Equivalently to the Christoffel tree, the directive sequence of the Christoffel word of slope $\frac{a}{b}$ can be read in the SB-tree by using the terms of the continued fraction representation of $\frac{a}{b}$. The following lemma presents this connection (a proof can be found in [9] and [12]). 
Lemma 1. [9] Let $w$ be the Christoffel word of slope $\frac{a}{b}$ whose continued fraction representation is $\left[a_{0}, a_{1}, \ldots, a_{z}\right]$. The directive sequence of $w$ has the following form: $\Delta\left(\frac{a}{b}\right)=1^{a_{0}} 0^{a_{1}} 1^{a_{2}} \ldots p^{a_{z}-1}$, where $p \in\{0,1\}$ according to the parity of $z$.

Referring again to the continued fraction representation $[0,1,2,2]$ of $\frac{5}{7}$, the directive sequence $\Delta\left(\frac{5}{7}\right)$ is exactly $1^{0} 0^{1} 1^{2} 0^{1}$.

\subsection{The Second Order Balance Matrix}

The second order of balancedness of a Christoffel word $w$, denoted $\delta^{2}(w)$, provides an idea of how uniformly the factors of each abelian class, as defined in [6], are distributed in $w$. It is the maximal value of the second order balance matrix, denoted $U_{w}$, introduced in [12]. In order to construct this matrix, the author had to define the balance matrix $B_{w}$, of a binary word $w$. This matrix calculates the order of balancedness, that is usually obtained by computing $\left.|| u\right|_{1}-|v|_{1} \mid \leq k$ for any factors $u$ and $v$ of same length, of any binary word $w=w_{1} w_{2} \ldots w_{n}$ in an explicit way. To do that, we first compute the matrix $S_{w}$ of dimension $(n-1) \times n$ whose generic element $S_{w}[i, j]$ counts the number of elements 1 in the $i$-length prefix of the conjugate of $w$ starting in position $j$. We let $M[i]$ represents the $i^{t h}$ row of any matrix M.

The balance matrix $B_{w}$ is computed from $S_{w}$ by subtracting the minimum of a given row to each of its element, i.e.,

$$
B_{w}[i, j]=S_{w}[i, j]-\min \left\{S_{w}[i]\right\} .
$$

By construction, we can note the following lemma:

Lemma 2. Let $w=w_{1} w_{2} \ldots w_{n}$, the maximum element of $B_{w}$ is equal to the balance orer of $w$.

We denote the balance order by $\delta(w)$. A simple example will clarify the construction.

Example 1. Let us consider the Christoffel word $w=00100101$ of slope $\frac{3}{5}$. We provide the matrices $S_{w}$ and $B_{w}$ :

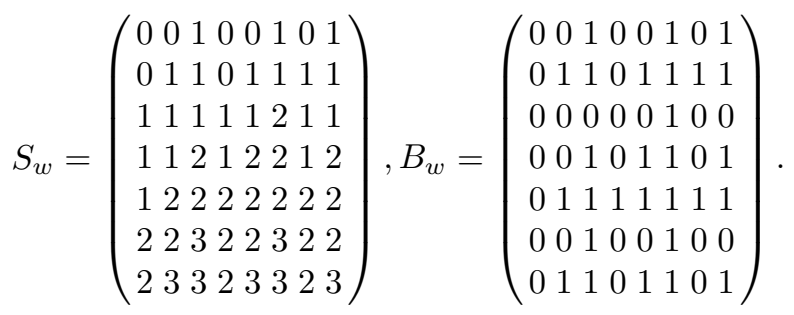

Since each Christoffel word $w$ is 1-balanced, then the rows of its balance matrix $B_{w}$ are binary words too. So, we can push the notion of balancedness to a second level by studying the balancedness of the rows of $B_{w}$. Let us define the second order balance matrix $U_{w}$ of dimension $(n-1) \times(n-1)$, with $n=|w|$, as $U_{w}[i, j]=\max \left(B_{B_{w}[i]}[j]\right)$. In words, the element $U_{w}[i, j]$ shows the balancedness of the $i$-th row of $B_{w}$ with respect to its proper factors of length $j$. More details and properties about this matrix can be found in [12]. 
Example 2. Let us consider the 1-balanced word $w$ of Example 1. The fourth row of the matrix $U_{w}$ is obtained by computing the balance value of the fourth row of $B_{w}$ with respect to all the possible factors' lengths from 1 to 8 . We explicitly determine the element $U_{w}[4,3]$ : the eight factors of the conjugates of $B_{w}[4]$ of length 3 are $(001,010,101,011,110,101,101,100)$, and the maximum difference of the number of elements 1 between any two of them is 1 , so $U_{w}[4,3]=1$. Doing an analogous computation for all the lengths from 1 to 7 , we obtain $U_{w}[4]=(1,2,1,2,1,2,1)$.

So, the second order of balancedness of a Christoffel word $w$ provides an idea of how uniformly the factors of each abelian class, as defined in [6], are distributed in $w$.

\section{Path Minimality in SB-Tree}

In this section, we prove some new properties of the second order balance parameter for Christoffel words and we show how it is distributed on the Christoffel tree. Finally, we characterize the paths of the tree where the parameter's growth is minimal. So, let us consider the following two ancestors of $\frac{a}{b}=\left[a_{0}, \ldots, a_{z}\right]$ in the SB-tree:

$\frac{u}{v}$ whose continued fraction expansion is $=\left[a_{0}, \ldots, a_{z-1}+1\right]$

$\frac{v}{\theta}$ whose continued fraction expansion is either $\left[a_{0}, \ldots, a_{z}-2\right]$ if $a_{z}>2$, or $\left[a_{0}, \ldots, a_{z-2}\right]$ if $a_{z}=2$.

In [12], Section 6.1, the authors proved that the matrix $U_{w}$, with $w=C\left(\frac{a}{b}\right)$, can be decomposed into blocks belonging either to $U_{w_{1}}$ or to $U_{w_{2}}+1$, with $w_{1}=C\left(\frac{u}{v}\right)$ and $w_{2}=C\left(\frac{\rho}{\theta}\right)$. From this result it immediately follows:

Theorem 1. The second order balance value of $C\left(\frac{a}{b}\right)$ is:

$$
\delta^{2}\left(C\left(\frac{a}{b}\right)\right)=\max \left(\delta^{2}\left(C\left(\frac{u}{v}\right)\right), \delta^{2}\left(C\left(\frac{\rho}{\theta}\right)\right)+1\right) .
$$

Using the previous result, we set a lower bound to the growth of the second order balanced value inside the Christoffel tree. The next lemma states that $\delta^{2}\left(C\left(\frac{a}{b}\right)\right)$ increases according to the distance between $\frac{a}{b}$ and $\frac{u}{v}$ in the SB-tree.

Lemma 3. Let $\frac{a}{b}=\left[a_{0}, \ldots, a_{z}\right]$ and belongs to the level $k$ on the SB-tree, where $\delta^{2}\left(C\left(\frac{u}{v}\right)\right)=t$ and $\delta^{2}\left(C\left(\frac{\rho}{\theta}\right)\right)=n$.

i) If $a_{z}=2$, then $\delta^{2}\left(C\left(\frac{a}{b}\right)\right) \in\{t ; t+1\}$.

ii) If $a_{z}>2$, then $\delta^{2}\left(C\left(\frac{a}{b}\right)\right)=n+1$, i.e it is increased by one value each 2 levels after $\frac{u}{v}$.

Proof. Let us consider these two rational numbers $\frac{a}{b}=\left[a_{0}, \ldots, a_{z}\right]$ and $u / v=$ $\left[a_{0}, \ldots, a_{z-1}+1\right]$, where $\delta^{2}\left(C\left(\frac{u}{v}\right)\right)=t$.

If $a_{z}=2$, then $\frac{\rho}{\theta}=\left[a_{0}, \ldots, a_{z-2}\right]$, in this case, its second order value is equal to $n \leq t$. In all the cases, from Theorem $1, \delta^{2}\left(C\left(\frac{a}{b}\right)\right)=\max \left(\delta^{2}\left(C\left(\frac{u}{v}\right)\right), \delta^{2}\left(C\left(\frac{\rho}{\theta}\right)\right)+1\right)$ that can be either $t$ or $t+1$.

If $a_{z} \geq 3$, then we must consider two cases for the rational number $\frac{\rho}{\theta}$. 
- If $a_{z}=3$, in this case $\frac{\rho}{\theta}=\frac{u}{v}$ and $n=t$, then: $\delta^{2}\left(C\left(\frac{a}{b}\right)\right)=\max \left(\delta^{2}\left(C\left(\frac{u}{v}\right)\right), \delta^{2}\left(C\left(\frac{\rho}{\theta}\right)+1\right)=n+1\right.$.

- If $a_{z} \geq 4$, in this case $\frac{\rho}{\theta}=\left[a_{0}, a_{1}, \cdots, a_{z}-2\right]$, and $n \geq t$ then: $\delta^{2}\left(C\left(\frac{a}{b}\right)\right)=\delta^{2}\left(C\left(\frac{\rho}{\theta}\right)\right)+1=n+1$.

Finally, we provide a lower bound, denoted by $\delta_{k}^{2}$, for the second order balance value of any element at level $k$ in the Christoffel tree.

Theorem 2. For each level $k$ in the $S B$ tree, we have: $\delta_{k}^{2} \geq\left\lceil\frac{k}{3}\right\rceil$.

Proof. By induction on the levels of the Stern-Brocot tree, we have $\delta_{1}^{2}=\delta_{2}^{2}=1$; and $\delta_{3}^{2} \in\{1,2\}$ while $\delta_{4}^{2}=2$. Hence the minimal value of $\delta_{3}^{2}$ (denoted $\min \delta_{3}^{2}$ ) is equal to 1 and $\min \delta_{4}^{2}=2$. Which means that $\delta_{i}^{2} \geq\left\lceil\frac{i}{3}\right\rceil$ with $1 \leq i \leq 4$.

Suppose that it is true for all the levels till $k=3 k^{\prime}$; i.e $\min \delta_{k}^{2}=\min \delta_{k-1}^{2}=$ $\min \delta_{k-2}^{2}=k^{\prime}$. We prove that $\delta_{k+1}^{2} \geq\left\lceil\frac{k+1}{3}\right\rceil=k^{\prime}+1$.

All the children of the fractions on level $k$, with $\delta^{2}>k^{\prime}$, have $\delta^{2} \geq k^{\prime}+1$. It remains to study the case where $\delta_{k}^{2}=k^{\prime}$. For that, we let $\frac{u}{v}$ be a fraction at level $k$ with $\delta^{2}\left(C\left(\frac{u}{v}\right)\right)=k^{\prime}$. By contradiction, we let $\frac{c}{d}$ be the fraction at level $k+1$ with $\delta^{2}\left(C\left(\frac{c}{d}\right)\right)=k^{\prime}$. This fraction is either in the same, or opposite direction of $\frac{u}{v}$. If $\frac{c}{d}$ is in the same direction of $\frac{u}{v}$, then by Lemma 3, we get $\delta^{2}\left(C\left(\frac{a}{b}\right)\right)=k^{\prime}-1$, where $\frac{a}{b}$ is the fraction at level $k-1$. If $\frac{c}{d}$ is in the opposite direction, then the fraction $\frac{u}{v}$ can be either in the same direction or opposite to $\frac{a}{b}$. In this case, we let $\frac{m}{n}$ be the fraction at level $(k-2)$ and we have:

- If $u / v=(a / b)$ then by Lemma $3, \delta^{2}\left(C\left(\frac{m}{n}\right)\right)=k^{\prime}-1$.

- If $u / v=(a / b)$, then if $\delta^{2}\left(C\left(\frac{a}{b}\right)\right)=k^{\prime}$, we get: $\delta^{2}\left(C\left(\frac{c}{d}\right)\right)=\max \left(\delta^{2}\left(C\left(\frac{u}{v}\right)\right), \delta^{2}\left(C\left(\frac{m}{n}\right)\right)+1\right)=k^{\prime}$. If $\delta^{2}\left(C\left(\frac{m}{n}\right)\right)=k^{\prime}$ then $\delta^{2}\left(C\left(\frac{c}{d}\right)\right)=k^{\prime}+1$; and if $\delta^{2}\left(C\left(\frac{m}{n}\right)\right)=k^{\prime}-1$ we get a contradiction since $\delta_{(k-2)}^{2} \geq\left\lceil\frac{k-2}{3}\right\rceil=k^{\prime}$.

Therefore $\delta^{2} \geq k^{\prime}+1$ for all the fractions on the level $k+1$ thus

$$
\delta_{k+1}^{2} \geq\left\lceil\frac{k+1}{3}\right\rceil=\left\lceil\frac{3 k^{\prime}+1}{3}\right\rceil=k^{\prime}+1 .
$$

The remaining part of the section, is devoted to the study of the paths of the SB-tree whose related Christoffel words realize the lower bound. The symmetry of the tree allows us to consider, without loss of generality, its left part only, i.e., those fractions less than or equal to 1 and whose continued fractions have $a_{0}=0$. We denote the subtree $S B_{L}$.

\subsection{Minimal Paths in the SB-Tree}

Let us assign to each fraction of $S B_{L}$ the $\delta^{2}$ value of its related Christoffel word starting from the root $\frac{1}{1}$ : Fig. 4 shows the computation till the fifth level of the tree. 


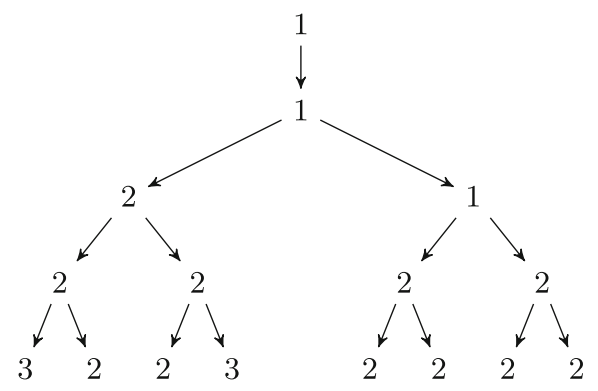

Fig. 4. The second order balancedness of the rational numbers belonging to the first five levels of $S B_{L}$.

Let us indicate by $P\left(\frac{a}{b}\right)$ the sequence of all the fractions in the path from the root of $S B_{L}$ to the element $\frac{a}{b}$, and by $P_{\delta^{2}}\left(\frac{a}{b}\right)$ the sequence of the related $\delta^{2}$ values. From Lemma 3 , it follows that $P_{\delta^{2}}\left(\frac{a}{b}\right)$ is a weakly increasing integer sequence.

As an example, let us consider the fraction $\frac{7}{12}$, lying in the sixth level of $S B_{L}$. We have $P\left(\frac{7}{12}\right)=\left(\frac{1}{1}, \frac{1}{2}, \frac{2}{3}, \frac{3}{5}, \frac{4}{7}, \frac{7}{12}\right)$, and the related $\delta^{2}$ sequence is $P_{\delta^{2}}\left(\frac{7}{12}\right)=$ $(1,1,1,2,2,2)$.

We give now the definition of a minimal path at a certain level $k$ of $S B_{L}$.

Definition 1. For each level $k$ of the $S B_{L}$, the minimal path $M P_{k}$ is given by: $\left(1,1,1,2,2,2,3, \ldots,\left\lceil\frac{k}{3}\right\rceil\right)$.

In order to give an example, we consider the Fibonacci sequence $\left\{f_{k}\right\}_{k=0}^{\infty}=$ $1,1,2,3,5,8,13, \ldots$, and define the $z i g$-zag path to be the sequence of rational numbers obtained by the ratio of two consecutive elements. It is well known that the golden number is the limit of the sequence of the rational numbers $\frac{f_{k}}{f_{k+1}}$, with $k \geq 0$. The continued fraction representations of these rational numbers are of the form $[0,1,1 \ldots, 1,2]$, and they constitute a (infinite) path in $S B_{L}$. This path realizes the lower bound stated in Theorem 2 assuring that, for each $k, P_{\delta^{2}}\left(\frac{f_{k}}{f_{k+1}}\right)$ is minimal, and it equals $M P_{k}$.

Now we focus our study on the questions: For each level $k$, is the $z i g$-zag path the only minimal one? If no, can we characterize all the minimal paths in $S B_{L}$ ?

\subsection{A Connection Between $\delta^{2}$ Value and the Continued Fraction Representation}

Let us consider the following continued fraction representations:

$$
\begin{aligned}
& \frac{a}{b}=\left[a_{0}, a_{1}, \cdots, a_{z}\right] ; \frac{u}{v}=\left[a_{0}, a_{1}, \cdots, a_{z-1}+1\right] ; \frac{p}{q}=\left[a_{0}, \cdots, a_{z-2}+1\right] ; \\
& \frac{s}{t}=\left[a_{0}, \cdots, a_{z-2}\right] ; \text { and } \frac{\rho}{\theta}= \begin{cases}{\left[a_{0}, a_{1}, \cdots, a_{z}-2\right]} & \text { if } a_{z} \geq 4 \\
{\left[a_{0}, a_{1}, \cdots, a_{z-1}+1\right]} & \text { if } a_{z}=3 \\
{\left[a_{0}, a_{1}, \cdots, a_{z-2}\right]} & \text { if } a_{z}=2 .\end{cases}
\end{aligned}
$$


From Lemma 3, we know that $\delta^{2}\left(C\left(\frac{a}{b}\right)\right)$ is related to $\delta^{2}\left(C\left(\frac{u}{v}\right)\right)$ and $\delta^{2}\left(C\left(\frac{\rho}{\theta}\right)\right)$. In the final part of the section, we will show how this relation reflects on their continued fraction representations.

Theorem 3. Let $\frac{a}{b}=\left[a_{0}, a_{1}, \cdots, a_{z}\right]$, we have:

$$
\delta^{2}\left(C\left(\frac{a}{b}\right)\right)=\left\{\begin{array}{lr}
\delta^{2}\left(C\left(\frac{u}{v}\right)\right) & \text { if } a_{z-1} \geq 2 \text { and } a_{z}=2 \\
\delta^{2}\left(C\left(\frac{\rho}{\theta}\right)\right)+1 & \text { elsewhere. }
\end{array}\right.
$$

Proof. Without loss of generality and in order to enlighten the notation, we denote $\delta^{2}\left(C\left(\frac{a}{b}\right)\right)$ by $D_{a}, \delta^{2}\left(C\left(\frac{u}{v}\right)\right)=D_{u}, \delta^{2}\left(C\left(\frac{\rho}{\theta}\right)\right)=D_{r}, \delta^{2}\left(C\left(\frac{p}{q}\right)\right)=D_{p}$ and $\delta^{2}\left(C\left(\frac{s}{t}\right)\right)=D_{s}$. Equivalently, by symmetry, we consider $z$ to be an even number which means that the last element of $\Delta\left(\frac{a}{b}\right)$ is equal to 1 . We start by considering separately the cases $a_{z} \geq 4$ and $a_{z}=3$, then we discuss the case $a_{z}=2$.

1. If $a_{z} \geq 4$, we let $D_{u}=d$, then $D_{r} \geq d$ since $\frac{\rho}{\theta}>\frac{u}{v}$. Hence $D_{a}=\max \left(D_{u}, D_{r}+\right.$ 1) $=D_{r}+1$ (see Fig. 5 , on the left).

2. If $a_{z}=3$, in this case the fractions $\frac{u}{v}$ and $\frac{\rho}{\theta}$ are the same and we have: $D_{a}=\max \left(D_{u}, D_{r}+1\right)=\max \left(D_{r}, D_{r}+1\right)=D_{r}+1$ (see Fig. 5 , on the right).

3. If $a_{z}=2$, in this case we get several sub-cases depending on the values of $a_{z-1}$ and $a_{z-2}$.

(a) If $a_{z-1}=1$, we get 4 sub-cases that are summarized in the table of Fig. 6, where fraction $\frac{u}{v}=\left[a_{0}, \cdots, 2\right]$ and $D_{r}=d$. If $D_{p}=d$, then $D_{u}$ can be equal to $d$ or $d+1$. In the former case, it holds $D_{a}=\max \left(D_{u}, D_{r}+1\right)=$ $d+1=D_{r}+1$, while, in the latter, it holds then $D_{a}=\max (d+1, d+$ 1) $=d+1=D_{r}+1$. Acting similarly in the case $D_{p}=d+1$, it holds $D_{a}=d+1=D_{r}+1$.

(b) If $a_{z-1}=2$, the cases are represented in Fig. 7. If $D_{p}=d$, then by Lemma 3, $D_{u}=d+1$ and $D_{a}=d+1=D_{u}$. On the other hand, if $D_{p}=d+1$, then $D_{u}=d+2$ and $D_{a}=d+2=D_{u}$.

(c) If $a_{z-1}=3$, the rational numbers are represented in Fig. 8 where the fraction $\frac{k}{l}=\left[a_{0}, \cdots, a_{z-2}, 2\right]$ with $\delta^{2}\left(C\left(\frac{k}{l}\right)\right)=D_{k}$ must be considered. If $D_{k}=d$, by Lemma 3 , it holds $D_{u}=d+1$ and $D_{a}=d+1=D_{u}$, while if $D_{k}=d+1$, it holds $D_{u}=d+2$ and $D_{a}=d+2=D_{u}$.

(d) Finally, if $a_{z-1}>3$, it holds $D_{u}>D_{r}+1$ therefore, $D_{a}=D_{u}$.
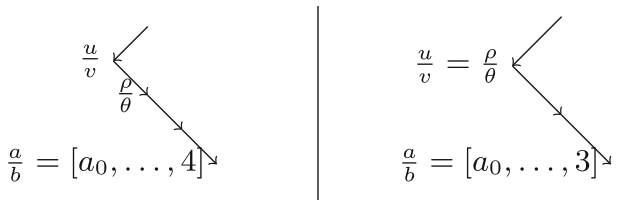

Fig. 5. Positions of the fractions $\frac{a}{b}, \frac{u}{v}, \frac{\rho}{\theta}$ on $S B_{L}$ in case where the last element of the continued fraction of $\frac{a}{b}=a_{z}=4$ or $a_{z}=3$ respectively. 
<smiles>CCCCC</smiles>

\begin{tabular}{|c|c|c|c|c|}
\hline$D_{r}$ & \multicolumn{4}{|c|}{$d$} \\
\hline$D_{p}$ & \multicolumn{2}{|c|}{$d$} & \multicolumn{2}{c|}{$d+1$} \\
\hline$D_{u}$ & $d$ & $d+1$ & $d+1$ & $d+1$ \\
\hline$D_{a}$ & $d+1$ & $d+1$ & $d+1$ & $d+1$ \\
\hline
\end{tabular}

Fig. 6. Position of the fractions $\frac{\rho}{\theta}, \frac{p}{q}, \frac{u}{v}$ and $\frac{a}{b}$, when $a_{z}=2$, and $a_{z-1}=1$, and the table showing the 4 possible subcases

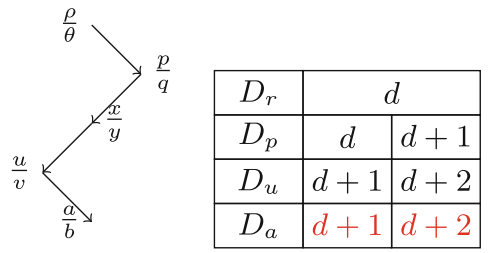

Fig. 7. Position of the fractions $\frac{\rho}{\theta}, \frac{p}{q}, \frac{u}{v}$ and $\frac{a}{b}$, when $a_{z}=2$, and $a_{z-1}=2$ $a_{z}=2$ and the table representing the 2 sub-cases.

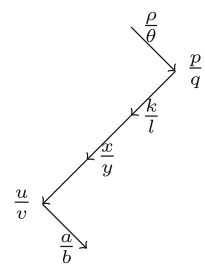

\begin{tabular}{|c|c|c|}
\hline$D_{r}$ & \multicolumn{2}{|c|}{$d$} \\
\hline$D_{k}$ & $d$ & $d+1$ \\
\hline$D_{u}$ & $d+1$ & $d+2$ \\
\hline$D_{a}$ & $d+1$ & $d+2$ \\
\hline
\end{tabular}

Fig. 8. Position of the fractions $\frac{\rho}{\theta}, \frac{p}{q}, \frac{k}{l}, \frac{u}{v}$ and $\frac{a}{b}$, when $a_{z}=2$, and $a_{z-1}=3$ and the table representing the 2 sub-cases.

\subsection{General Form of a Minimal Path}

In this section we give the final result of the paper, by providing a characterization on the elements of the continued fraction of $\frac{a}{b}$ in order to consider $P_{\delta^{2}}\left(\frac{a}{b}\right)$ as a minimal path in $S B_{L}$.

At level 4 of the $S B_{L}$, only two rational numbers out of 4 belong to a minimal path as we can see in Table 1 . We have $P_{\delta^{2}}\left(\frac{3}{5}\right)=P_{\delta^{2}}\left(\frac{3}{4}\right)=(1,1,1,2)$, where $\frac{3}{5}=[0,1,1,2]$ and $\frac{3}{4}=[0,1,3]$. This insures that the continued fraction of any rational number in $S B_{L}$, who belongs to a minimal path must starts with $[0,1]$. At level 5 , we note that these two rational numbers generate 4 rational numbers that belong to a minimal path. While at level 6 , we get again 4 rational numbers that are also represented in Table 1. By Definition 1, we can notice that three consecutive elements $p_{i}, p_{i+1}$, and $p_{i+2}$, of a minimal path are such that $p_{i+1}-p_{i} \leq 1$. Hence, all the children of the rational numbers of $S B_{L}$ belonging to $M P_{6}$ maintain the minimality at levels 7 and 8 . While at level 9 , there exist only $16\left(=4^{2}\right)$ fractions that belong to $M P_{9}$ and are represented in Table 2.

Considering these first levels, we note that the continued fraction representations of the elements of $M P_{9}$ must start with 0,1 and end with 2 . On the other hand, all the remaining $\delta^{2}$ values of each element of $M P_{9}$ can be grouped into the blocks $(1,1,1) ;(1,2) ;(2,1)$ or 3 as the red and blue colored sequences in Table 2 witness. Aware of this, we can characterize the elements of $S B_{L}$ that belongs to a minimal path. 
Table 1. The rational numbers $\frac{a}{b}$ at level 4 of $S B_{L}$ with their continued fraction representation, $P\left(\frac{a}{b}\right)$ and $P_{\delta^{2}}\left(\frac{a}{b}\right)$. The four rational numbers at level 6 that belong to a minimal path with their continued fraction representation, and sequences $P$ and $P_{\delta^{2}}$.

\begin{tabular}{|c|c|c|c|c|}
\hline Level & Fraction $\frac{a}{b}$ & Continued fraction & $P\left(\frac{a}{b}\right)$ & $P_{\delta^{2}}\left(\frac{a}{b}\right)$ \\
\hline 4 & $\frac{1}{4}$ & {$[0,4]$} & $\left(\frac{1}{1}, \frac{1}{2}, \frac{1}{3}, \frac{1}{4}\right)$ & $(1,1,2,2)$ \\
\hline 4 & $\frac{2}{5}$ & {$[0,2,2]$} & $\left(\frac{1}{1}, \frac{1}{2}, \frac{1}{3}, \frac{2}{5}\right)$ & $(1,1,2,2)$ \\
\hline 4 & $\frac{3}{5}$ & {$[0,1,1,2]$} & $\left(\frac{1}{1}, \frac{1}{2}, \frac{2}{3}, \frac{3}{5}\right)$ & $(1,1,1,2)$ \\
\hline 4 & $\frac{3}{4}$ & {$[0,1,3]$} & $\left(\frac{1}{1}, \frac{1}{2}, \frac{2}{3}, \frac{3}{4}\right)$ & $(1,1,1,2)$ \\
\hline 6 & $\frac{8}{13}$ & {$[0,1,1,1,1,2]$} & $\left(\frac{1}{1}, \frac{1}{2}, \frac{2}{3}, \frac{3}{5}, \frac{5}{8}, \frac{8}{13}\right)$ & $(1,1,1,2,2,2)$ \\
\hline 6 & $\frac{8}{11}$ & {$[0,1,2,1,2]$} & $\left(\frac{1}{1}, \frac{1}{2}, \frac{2}{3}, \frac{3}{4}, \frac{5}{7}, \frac{8}{11}\right)$ & $(1,1,1,2,2,2)$ \\
\hline 6 & $\frac{7}{12}$ & {$[0,1,1,2,2]$} & $\left(\frac{1}{1}, \frac{1}{2}, \frac{2}{3}, \frac{3}{5}, \frac{4}{7}, \frac{7}{12}\right)$ & $(1,1,1,2,2,2)$ \\
\hline 6 & $\frac{7}{9}$ & {$[0,1,3,2]$} & $\left(\frac{1}{1}, \frac{1}{2}, \frac{2}{3}, \frac{3}{4}, \frac{4}{5}, \frac{7}{9}\right)$ & $(1,1,1,2,2,2)$ \\
\hline
\end{tabular}

Table 2. The 16 rational numbers that belong to $M P_{9}$ on $S B_{L}$.

\begin{tabular}{|c|c|c|c|}
\hline$\frac{29}{50}$ & $\frac{27}{46}$ & $\frac{26}{45}$ & $\frac{23}{39}$ \\
\hline$[0,1, \mathbf{1}, \mathbf{2}, 1,1,1,2]$ & {$[0,1, \mathbf{1}, \mathbf{2}, 2,1,2]$} & {$[0,1, \mathbf{1}, \mathbf{2}, 1,2,2]$} & {$[0,1, \mathbf{1}, \mathbf{2}, 3,2]$} \\
\hline$\frac{34}{55}$ & $\frac{30}{49}$ & $\frac{31}{50}$ & $\frac{25}{41}$ \\
\hline$[0,1, \mathbf{1}, \mathbf{1 , 1}, 1,1,1,2]$ & {$[0,1, \mathbf{1}, \mathbf{1}, \mathbf{1}, 2,1,2]$} & {$[0,1, \mathbf{1 , 1} \mathbf{1}, 1,2,2]$} & {$[0,1, \mathbf{1 , 1 , 1 , 3 , 2 ]}$} \\
\hline$\frac{34}{47}$ & $\frac{30}{41}$ & $\frac{31}{43}$ & $\frac{25}{34}$ \\
\hline$[0,1, \mathbf{2 , 1}, 1,1,1,2]$ & {$[0,1, \mathbf{2 , 1}, 2,1,2]$} & {$[0,1, \mathbf{2 , 1}, 1,2,2]$} & {$[0,1, \mathbf{2 , 1}, 3,2]$} \\
\hline$\frac{29}{37}$ & $\frac{27}{35}$ & $\frac{26}{33}$ & $\frac{23}{30}$ \\
\hline$[0,1, \mathbf{3}, 1,1,1,2]$ & {$[0,1, \mathbf{3}, 2,1,2]$} & {$[0,1, \mathbf{3}, 1,2,2]$} & {$[0,1, \mathbf{3}, 3,2]$} \\
\hline
\end{tabular}

Theorem 4. Let $\frac{a}{b}$ be an element of $S B_{L}$ at a certain level $k \geq 6$ and whose continued fraction representation is $\left[a_{0}, a_{1}, \ldots, a_{z}\right]$. If the $a_{i}$ 's respect the following conditions: $a_{0}=0, a_{1}=1, a_{z}=2$ and the elements $a_{2}, \ldots, a_{z-1}$ are obtained by the concatenation of the blocks $(1,1,1),(2,1)(1,2)$ and $(3)$, then $\frac{a}{b} \in M P_{k}$.

Proof. By the computation of the first minimal paths of $S B_{L}$ we have that $a_{0}=0$ and $a_{1}=1$. From Lemma 3, we know that $a_{z}$ must be equal to 2 in order to realize the minimal growth. Now, from Definition 1 , we know that the rational number $\frac{a}{b}$ belongs to a minimal path if the values of $P_{\delta^{2}}\left(\frac{a}{b}\right)$ are increased by 1 , each three steps. Relying on that, we consider all the possible ways to pass from a level $t$ to a level $t+3$ in $S B_{L}$ keeping the minimal growth of one, and we easily realize that these elements can only have one the following forms:

$(1,1,1),(2,1)(1,2)$ or $(3)$, i.e. all the possible integer decompositions of the number three. Note that the first case, is equivalent to the zig-zag path, while the other cases are obtained by Theorem 3 and Lemma 3 .

Finally, some simple computations lead to the following: 
Corollary 1. Let $k$ be a certain level in $S B_{L}$, the number of $M P_{k}$ is equal to:

$$
\begin{cases}4^{\frac{k}{3}-1} & \text { if } k \equiv_{3} 0 \\ 2 \cdot 4^{\left\lfloor\frac{k}{3}\right\rfloor-1} & \text { if } k \equiv_{3} 1 \\ 4 \cdot 4^{\left\lfloor\frac{k}{3}\right\rfloor-1} & \text { if } k \equiv_{3} 2\end{cases}
$$

This last theorem determines the form of the minimal paths inside the SBtree according to the $\delta^{2}$-paths, and from a geometrical perspective, it defines a new family of Christoffel words that deserves to be investigated. A further generalization of the order of balancedness may also identify special paths of the SB-tree showing a fixed point property.

\section{References}

1. Berstel, J., De Luca, A.: Sturmian words, Lyndon words and trees. Theor. Comput. Sci. 178(1), 171-203 (1997)

2. Berstel, J., Lauve, A., Reutenauer, C., Saliola, F.: Combinatorics on words: Christoffel words and repetition in words, Université de Montréal et American Mathematical Society (2008)

3. Borel, J.-P., Laubie, F.: Quelques mots sur la droite projective réelle. Journal de théorie des nombres de Bordeaux 5(1), 23-51 (1993)

4. Brocot, A.: Calcul des rouages par approximation, Revue chronométrique. Journal des horlogers, scientifique et pratique 3, 186-194 (1861)

5. Lothaire, M.: Algebraic Combinatorics on Words, Encyclopedia of Mathematics and its Applications, vol. 90. Cambridge University Press, Cambridge (2002)

6. Richomme, G., Saari, K., Zamboni, L.-Q.: Abelian properties of words, arXiv preprint arXiv:0904.2925 (2009)

7. Fraenkel, A.: The bracket function and complementary sets of integers. Can. J. Math. 21, 6-27 (1969)

8. Fraenkel, A., Levitt, J., Shimshoni, M.: Characterization of the set of values $f(n)=$ $[n a], n=1,2, \ldots$. Discrete Math. 2(4), 335-345 (1972)

9. Graham, R.E., Knuth, D.E., Patashnik, O.: Concrete Mathematics. A Foundation for Computer Science, 2nd edn. Addison-Wesley, Reading (1994)

10. Pettofrezzo, A.J., Byrkit, D.R.: Elements of Number Theory. Prentice Hall, Englewood Cliffs (1970)

11. Stern, M.A.: Ueber eine zahlentheoretische Funktion. Journal für die reine und angewandte Mathematik 55, 193-220 (1858)

12. Tarsissi, L., Vuillon, L.: Second order balance property on Christoffel words. In: Slamanig, D., Tsigaridas, E., Zafeirakopoulos, Z. (eds.) MACIS 2019. LNCS, vol. 11989, pp. 295-312. Springer, Cham (2020). https://doi.org/10.1007/978-3-03043120-4_23 